\title{
The Use of Taguchi Method in Design of Dies Propeller Production Using CNC Machine
}

\author{
Edi Jadmiko ${ }^{1}$, Purwadi Wahyunugroho ${ }^{2}$ \\ (Received: 01 August 2019 / Revised: 24 November 2019 / Accepted: 28 November 2019)
}

\begin{abstract}
A speed boat's propeller moves it forward by spinning against the water. Propellers use the laws of physics to propel, or drive forward, an aircraft or a boat. The basic structure of a propeller is a spinning or rotating shaft with wide, curved blades attached to it. The very simplest propellers were first used in ancient Greece, where the inventor Archimedes invented a "screw propeller" that moved water for irrigating crops. A method for making propeller is to use a casting method with sand mold. In operating, the sand mold can only be used for one use, and in the molding process must be reconstructed, which of course, requires cost and additional time, so it is less efficient. Therefore, making a propeller with a permanent mold is the right solution. In this study, the design of the dies propeller production process with CNC (Computerized Numerical Control) machine was designed using the MasterCam software simulation method. In addition, variations in machining parameters feed rate, retract rate, and depth of cut were also carried out using the Taguchi method. The research has known the step of dies propeller production process and the time for cutting that used to calculate the most efficient variable variation. Based on the simulation that has been done, for making dies propeller requires ten steps of processing, there are facing, pocket, contour (top die), rough surface pocket, surface finish contour (top die), surface finish parallel, contour (bottom die), and surface finish contour (bottom die). From a series of work processes designed, the total processing time was produced for top die $\mathbf{7 2 9 , 1 7}$ minutes, and cutting power was $9,734 \mathrm{kWh}$, and for the bottom, die is about 1329,65 minutes, and cutting power was $14,543 \mathrm{kWh}$.
\end{abstract}

Keywords-CNC machines, machining parameters, mastercam, permanent mold, propeller, simulation, taguchi method.

\section{INTRODUCTION}

$\mathrm{M}$ anufacturing industry activities often deal with increasing customer demand. Manufacturing activities are required to develop production methods continue to be more competitive in making products such as sustainable change and increasing the flexibility of the production system. These developments have the main goal of getting a shorter production time, cheaper products, and the best performance of the product. An example of the manufacturing industry is the metal casting industry, and the products produced are propeller.

In the process of casting metal, there are several kinds of molds. Molds are often used are permanent mold (metal molds) and non-permanent mold (sand molds). In general, the metal casting industry uses sand mold (sand casting). In operating sand molds are made manually so that the dimensions are less precise and can only be used for one use and in the moulding process must be reconstructed which of course, requires cost and extra time[1].

Permanent mold (metal mold) is a type of mold that can be used repeatedly, and this mold is usually made of metal material. Using metal molds will produce cast products that are more economical both in quality and quantity. With this permanent mold, even though the initial investment is relatively more expensive, for more production, the use of metal molds will be profitable, and this mold can be used repeatedly. The technology that can be used to produce permanent molds for the

${ }^{1}$ Edi Jadmiko, Department of Marine Engineering, Institut Teknologi Sepuluh Nopember, Surabaya, 60111, Indonesia. E-mail: e.djatmiko@yahoo.com

${ }^{2}$ Purwadi Wahyunugroho, Department of Marine Engineering, Institut Teknologi Sepuluh Nopember, Surabaya, 60111, Indonesia. Email: Purwadi.wahyunugroho18@gmail.com propeller is CNC (Computerized Numerical Control) machines [2].

Software used for CNC programming is the MasterCam software. By using MasterCam software, a programmer only needs to draw an object image and plan the machining parameters to be used. After that, the instructions for the process of working the object will be made by the software. These commands will be converted into the numerical language (G-Code), which can be understood by $\mathrm{CNC}$ machines and then transferred to $\mathrm{CNC}$ machines for execution. But before executing the command directly on a $\mathrm{CNC}$ machine, MasterCam software can execute the command in the form of a simulation aluminum so that if there are errors, it can be identified. The simulation aims to reduce the risk of errors and work accidents when the G-code command is executed directly on a CNC machine [3].

Production costs are accumulated costs that occur during the production process of a material. Production cost are influenced by material costs, production processes, and other indirect costs that occur during the production process. This production cost calculation is used in the manufacturing industry to find out the true cost of making a machine component product so that it can be estimated how much profit will be obtained [4].

\section{METHOD}

\section{A. Permanent Die Casting}

Permanent die casting is the manufacture of metals with molds combined with hydrostatic pressure. This method is not practical for large casting and when using high boiling metals. Non-steel metals such as aluminium, zinc, tin, magnesium, bronze if made in this way the results are good. This mold consists of two or more parts 
combined with screws, clamps, plates or other removable tools after the product has hardened. In general, permanent molds are made from close-grain and clamped to one another. This mold is usually coated with heat-resisting wet mixture and soot, which will keep the mold from sticking and reduce the effect of cold on the metal [5].

\section{B. Propeller}

Propeller is a rounded blade that rotates in a circle, helping to move a vehicle by pushing against water or air. A speed boat's propeller moves it forward by spinning against the water. Propellers use the laws of physics to propel, or drive forward, an aircraft or a boat. The basic structure of a propeller is a spinning or rotating shaft with wide, curved blades attached to it. The very simplest propellers were first used in ancient Greece, where the inventor Archimedes invented a "screw propeller" that moved water for irrigating crops [6].

\section{CNC (Computerized Numerical Control)}

Computer Numerical Control, abbreviated as CNC is a machine tool automation system operated by abstractly programmed and stored commands on storage media, this is in contrast to a previous habit in which machine tools are usually controlled by hand spin or simple automation using cam[7]. The word NC itself is an English abbreviation of the word Numerical Control which means "numerical control". The first NC machines were first invented in the 1940s and 1950s, by modifying ordinary machine tools. In this case, ordinary machine tools are added with motors that will move the control wheels include the points entered into the system by the paper recorder. The blend of motor and mechanical servo was soon replaced by analog systems and then digital computers, creating a modern Machine Tool called the CNC Machine (computer numerical control) which later revolutionized the design process. Currently, CNC machines have a very close relationship with the CAD program. CNC machines are built to address the challenges in the modern manufacturing world. With a
CNC machine, the accuracy of a product can be guaranteed up to $1 / 100 \mathrm{~mm}$ more, mass product workmanship with exact same results molding and fast machining time [8].

\section{CAD/CAM Programming}

Computer-Aided Manufacturing (CAM) is a function of computer software to control the machine and its equipment in the work process of the object. CAM aims to make the production process faster, with more precise and consistent dimensions. CAM software is used in computer systems to facilitate programmers in producing complex contour and surface forms (machining of artistic products) without using complicated mathematical formula calculations. In the manufacturing process, a product generally begins with the design concept of the product, then proceeds with making a 3D model using a Computer-Aided Design (CAD) system. After modeling using a CAD system, the product model is transferred to the CAM system [9]. With the CAM system, a path will be made that the cutter (toolpath strategy) will pass through the $3 \mathrm{D}$ product that we made. The cutter path in the CAM system will later be used as a cutter path in the actual machining process in a $\mathrm{CNC}$ machine with the help of NC; post-processing is done on the cutter location (Cutter Location / CL data) where it defines the cutter path. With a post-processor, the CL data can be converted into a G-Code that can be read by CNC machines [10].

\section{E. Taguchi Method}

Taguchi method used in offline quality control activities at the design process stage of production, what is meant by offline quality control, is quality control activities in product design. In the Taguchi method, a matrix called orthogonal array is used to determine the number of minimal experiments that can give information as much as possible of all factors that influence the parameters. The most important part of orthogonal arrays lies in the selection of combinations level of input variables for each experiment[11].

TABLE 1.

ORTHOGONAL ARRAY 4 FACTORS WITH 3 LEVELS

\begin{tabular}{ccccc}
\hline $\begin{array}{c}\text { Experiment } \\
\text { no. }\end{array}$ & $\begin{array}{c}\text { Factor } \\
\text { A }\end{array}$ & $\begin{array}{c}\text { Factor } \\
\text { B }\end{array}$ & $\begin{array}{c}\text { Factor } \\
\text { C }\end{array}$ & $\begin{array}{c}\text { Factor } \\
\text { D }\end{array}$ \\
\hline 1 & 1 & 1 & 1 & 1 \\
2 & 1 & 2 & 2 & 2 \\
3 & 1 & 3 & 3 & 3 \\
4 & 2 & 1 & 2 & 3 \\
5 & 2 & 2 & 3 & 1 \\
6 & 2 & 3 & 1 & 2 \\
7 & 3 & 1 & 3 & 2 \\
8 & 3 & 2 & 1 & 3 \\
9 & 3 & 3 & 2 & 1 \\
\hline
\end{tabular}

\section{F. Analysis of Variance (ANOVA)}

Analysis of variance or ANOVA is a multivariate analysis technique that serves to differentiate the mean of more than two groups of data by comparing the variance. Analysis of variance is included in the statistical parametric category. As a parametric statistical tool, to be able to use the ANOVA formula must first be tested for assumptions including normality, heterogeneityand random sampling [13]. 
Analysis of variance can be used to analyze data from various types and research designs. Analysis of variance is widely used in many studies involving comparative testing, namely testing the dependent variable by comparing it to the independent sample groups observed [14].

\section{RESULT AND DISCUSSION}

In doing this thesis, a process must be prepared. There must be planning in the work so that it feels more focused and easier. In the discussion of this research will be discussed step by step, which will be carried out in the execution of the thesis.

\section{A. Model Design}

Making dies propeller models using existing data propeller. Modeling is done using SolidWorks software, which is then simulated using the Mastercam software to determine the total production time and cutting power requirement.

B. Machining Process Design (CAM) and Simulation

It was selecting and calculating $\mathrm{CNC}$ machining parameters needed, such as toolpath, tools, spindle speed, feed rate, and depth of cutfor the production ofDies Propeller. After determining the machining parameters of the process, a simulation (running) the MasterCam program is performed. It aims to look at the work results in each groove on each toolpath.

\section{Calculation of Total Time\& Power Requirement}

The total time of the production process is the overall processing time on the variations of the tool with the most optimal results and also the time of non-productive machinery. After obtaining the optimal time, we calculated also the power requirement for the work of the object.

D. Analysis of Varian (ANOVA)

Analysis of variance (ANOVA) statistics used to determine the effect of each factor variable (feed rate, retrack rate, and depth of cut) on the response variable (machining process power). The variances analysis process uses Minitab software 18.

A. Model Design

Workpieces that will be processed by $\mathrm{CNC}$ milling are dies propeller models. Below is the model of the dies propeller:

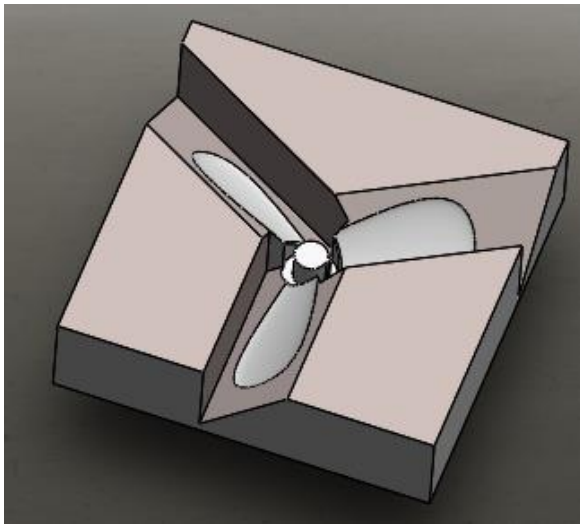

(a)

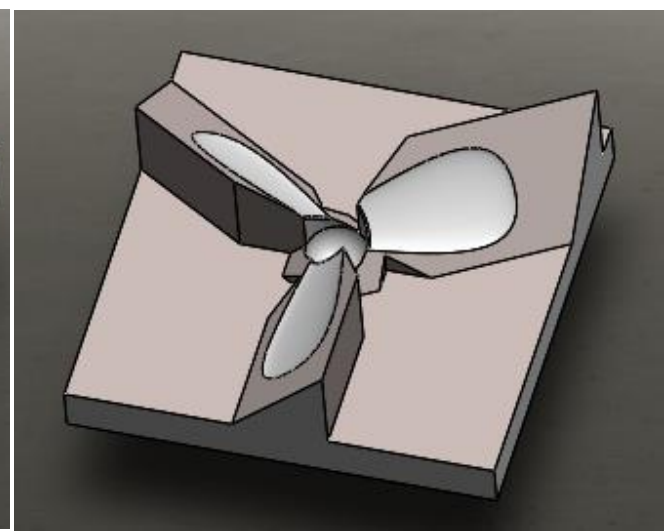

(b)

Figure 1. (a) Top Die (b) Bottom Die

\section{B. Mastercam Design Process}

The design of the machining process at MasterCam is the stage for determining the type of machining, the selection of boundaries or working area boundaries, and the selection of the right type of work for making dies propeller. In the MasterCam software, each type of work is called the toolpath. For dies propeller production process there are ten tool paths that is facing, pocket, contour (top die), rough surface pocket, surface finish contour (top die), multiaxis roughing, surface finish parallel, contour (bottom die), high-speed surface waterline, and surface finish contour (bottom die).

\section{Determination of Machining Parameters}

All machining parameters are calculated to get the engine optimization in the form of smoother finishes, more durable tools, and more optimal processing time and power. Machining parameters that need to be determined include:

a. Material type and tool diameter

b. Cutting speed c. Spindle speed

d. Feed rate and depth of cut

The formula for calculation machining parameters includes:

$\mathrm{CS}=\frac{\pi D N}{1000}, \mathrm{~N}=\frac{1000 C S}{\pi D}$

Where:

$\mathrm{CS}=$ Cutting Speed, $\mathrm{m} / \mathrm{min}$

$\mathrm{D}=$ Diameter tool, $\mathrm{mm}$

$\mathrm{N}$ = Spindle speed, $\mathrm{rpm}$

$\mathrm{F}=\mathrm{f}_{\mathrm{z}} \times \mathrm{N}$

Where:

$\mathrm{F}=$ Feed rate, $\mathrm{mm} / \mathrm{min}$

$\mathrm{f}_{\mathrm{z}} \quad=$ Cutting feed, $\mathrm{mm} / \mathrm{rev}$

$\mathrm{f}_{\mathrm{z}}=\mathrm{f}_{\mathrm{t}} \times \mathrm{Z}$ 
Where:

$\mathrm{f}_{\mathrm{t}} \quad=$ Cutting feed, $\mathrm{mm} / \mathrm{rev}$

$\mathrm{Z} \quad=$ Number of teeth/cutting edge
The results of setting machining parameters can be seen in Table 2 to Table 5 .

TABLE 2.

TOOLS SELECTION

\begin{tabular}{cccl}
\hline No & Tools & $\begin{array}{c}\text { Diameter } \\
(\mathrm{mm})\end{array}$ & Material \\
\hline 1 & Face End Mill & 75 & Carbida \\
2 & Flat End Mill & 20 & Carbida \\
3 & Flat End Mill & 25 & Carbida \\
4 & Sphere End Mill & 8 & Carbida \\
5 & Sphere End Mill & 20 & Carbida \\
\hline
\end{tabular}

TABLE 3.

SPINDLE SPEED CALCULATION

\begin{tabular}{cccc}
\hline No & Tools & $\begin{array}{c}\text { Diameter } \\
(\mathrm{mm})\end{array}$ & $\begin{array}{c}\text { Spindle Speed } \\
(\mathrm{Rpm})\end{array}$ \\
\hline 1 & Face End Mill & 75 & 389 \\
2 & Flat End Mill & 20 & 1457 \\
3 & Flat End Mill & 25 & 1457 \\
4 & Sphere End Mill & 8 & 3643 \\
5 & Sphere End Mill & 20 & 1457 \\
\hline
\end{tabular}

TABLE 4.

FEED RATE CALCULATION

\begin{tabular}{cccccc}
\hline & & Diameter & \multicolumn{2}{c}{ Feed Rate (mm/minute) } \\
No & Tools & $(\mathrm{mm})$ & Level I & Level II & Level III \\
\hline 1 & Face End Mill & 75 & 395 & 592 & 379 \\
2 & Flat End MIll & 20 & 592 & 1184 & 1776 \\
3 & Flat End Mill & 25 & 592 & 1184 & 1776 \\
4 & Sphere End Mill & 8 & 1110 & 2220 & 3331 \\
5 & Sphere End Mill & 20 & 592 & 1184 & 1776 \\
\hline
\end{tabular}

TABLE 5.

DEPTH OF CUT CALCULATION

\begin{tabular}{cccccc}
\hline \multirow{2}{*}{ No } & Process & \multicolumn{3}{c}{ Axial Depth of Cut (mm) } & \multirow{2}{*}{ Radial Depth of Cut (mm) } \\
& Facing & 1 & 1.5 & 2 & 45 \\
\hline 1 & Contour & 1 & 2 & 3 & 0.5 \\
2 & Surface Rough Pocket & 1 & 2 & 3 & 12 \\
3 & Surface Finish Contour & 0.5 & 0.75 & 1 & 0.5 \\
4 & Surface Finish Parallel & 1 & 2 & 3 & 12 \\
\hline 5 & & & & & \\
\hline
\end{tabular}

D. Calculation of Total Time \& Power Requirement

The calculation of time and power uses the Taguchi method with a variation of three-parameter combinations that is feed rate, retrack rate and depth of cut with three different levels then simulated in the Mastercam software to analyze the combination that produces the most optimum power response which is then used as a guideline for planning machining parameters. 
International Journal of Marine Engineering Innovation and Research, Vol. 5(1), Mar. 2020. 20-33 (pISSN:2541-5972, eISSN: 2548-1479)

TABLE 6.

PARAMETER VALUE OF FACING

\begin{tabular}{cccccc}
\hline Factor Name & Code & Level I & Level II & Level II & Unit \\
\hline Feed Rate & F & 395 & 592 & 790 & $\mathrm{~mm} / \mathrm{min}$ \\
Retrack Rate & $\mathrm{Cr}$ & 5000 & 7500 & 10000 & $\mathrm{~mm} / \mathrm{min}$ \\
Depth of Cut & $\mathrm{a}$ & 1 & 1.5 & 2 & $\mathrm{~mm}$ \\
\hline
\end{tabular}

TABLE 7.

POWER RESPONSES OF FACING

\begin{tabular}{ccccccccc}
\hline \multirow{2}{*}{ No } & \multicolumn{2}{c}{ Parameter Combination } & Time & MRR & HPc & HPm & \multirow{2}{*}{$\mathrm{kWh}$} \\
& $\mathrm{F}$ & $\mathrm{Vr}$ & $\mathrm{a}$ & $(\mathrm{min})$ & $\mathrm{mm} 3 / \mathrm{min}$ & $\mathrm{kW}$ & $\mathrm{kW}$ & \\
\hline 1 & 395 & 5000 & 1 & 60.3 & 17775 & 0.809 & 1.349 & 1.3553 \\
2 & 395 & 7500 & 1.5 & 56.03 & 26662.5 & 1.214 & 2.023 & 1.889 \\
3 & 395 & 10000 & 2 & 30.02 & 35550 & 1.618 & 2.697 & 1.3495 \\
4 & 592 & 5000 & 1.5 & 40.26 & 39960 & 1.819 & 3.032 & 2.0343 \\
5 & 592 & 7500 & 2 & 20 & 53280 & 2.425 & 4.042 & 1.3474 \\
6 & 592 & 10000 & 1 & 40.25 & 26640 & 1.213 & 2.021 & 1.3558 \\
7 & 790 & 5000 & 2 & 15.12 & 71100 & 3.237 & 5.394 & 1.3593 \\
8 & 790 & 7500 & 1 & 30.23 & 35550 & 1.618 & 2.697 & 1.3589 \\
9 & 790 & 10000 & 1.5 & 28.43 & 53325 & 2.427 & 4.046 & 1.917 \\
\hline
\end{tabular}

Table 6 shows the parameter value of the facing process. From table 7 it can be concluded that the optimal power response value in the facing process is in the $5^{\text {th }}$ combination with feed rate value of 592 $\mathrm{mm} /$ minute (level II), re-track rate $7500 \mathrm{~mm} /$ minute (level II), and depth of cut $2 \mathrm{~mm}$ (level III) with power response value $3,474 \mathrm{Wh}$ and time 20 minutes.

TABLE 8.

PARAMETER VALUE OF SURFACE ROUGH POCKET

\begin{tabular}{cccccc}
\hline Factor Name & Code & Level I & Level II & Level II & Unit \\
\hline Feed Rate & F & 592 & 1184 & 1776 & $\mathrm{~mm} / \mathrm{min}$ \\
Retrack Rate & Vr & 5000 & 7500 & 10000 & $\mathrm{~mm} / \mathrm{min}$ \\
Depth of Cut & a & 1 & 2 & 3 & $\mathrm{~mm}$ \\
\hline
\end{tabular}

TABLE 9.

POWER RESPONSES OF SURFACE ROUGH POCKET

\begin{tabular}{ccccccccc}
\hline \multirow{4}{*}{ No } & \multicolumn{3}{c}{ Parameter Combination } & Time & MRR & HPc & HPm & \multirow{2}{*}{ kWh } \\
& $\mathrm{F}$ & $\mathrm{Vr}$ & $\mathrm{a}$ & $(\mathrm{min})$ & $\mathrm{mm} 3 / \mathrm{min}$ & $\mathrm{kW}$ & $\mathrm{kW}$ & \\
\hline 1 & 592 & 5000 & 1 & 1043.18 & 7104 & 0.323 & 0.539 & 9.3707 \\
2 & 592 & 7500 & 2 & 527.52 & 14208 & 0.647 & 1.078 & 9.4772 \\
3 & 592 & 10000 & 3 & 366.8 & 21312 & 0.97 & 1.617 & 9.8847 \\
4 & 1184 & 5000 & 2 & 274.35 & 28416 & 1.294 & 2.156 & 9.8577 \\
5 & 1184 & 7500 & 3 & 190.6 & 42624 & 1.94 & 3.234 & 10.2727 \\
6 & 1184 & 10000 & 1 & 440.92 & 14208 & 0.647 & 1.078 & 7.9214 \\
7 & 1776 & 5000 & 3 & 131.65 & 63936 & 2.91 & 4.851 & 10.6433 \\
8 & 1776 & 7500 & 1 & 354.22 & 21312 & 0.97 & 1.617 & 9.5457 \\
9 & 1776 & 10000 & 2 & 180.3 & 42624 & 1.94 & 3.234 & 9.7176 \\
\hline
\end{tabular}

Table 8 shows the parameter value of the rough surface pocket. From table 9 it can be concluded that the optimal power response value in the surface rough pocket process is in the $6^{\text {th }}$ combination with feed rate value $1184 \mathrm{~mm} /$ minute (level II), re-track rate 10000 $\mathrm{mm} /$ minute(level III), and depth of cut $1 \mathrm{~mm}$ (level I) with power response value $7,9214 \mathrm{kWh}$ and processing time 440,92 minutes. 
International Journal of Marine Engineering Innovation and Research, Vol. 5(1), Mar. 2020. 20-33 (pISSN:2541-5972, eISSN: 2548-1479)

TABLE 10.

PARAMETER VALUE OF CONTOUR

\begin{tabular}{cccccc}
\hline Factor Name & Code & Level I & Level II & Level II & Unit \\
\hline Feed Rate & F & 592 & 1184 & 1776 & $\mathrm{~mm} / \mathrm{min}$ \\
Retrack Rate & $\mathrm{Vr}$ & 5000 & 7500 & 10000 & $\mathrm{~mm} / \mathrm{min}$ \\
Depth of Cut & $\mathrm{a}$ & 1 & 2 & 3 & $\mathrm{~mm}$ \\
\hline
\end{tabular}

TABLE 11.

POWER RESPONSES OF CONTOUR

\begin{tabular}{ccccccccc}
\hline \multirow{2}{*}{ No } & \multicolumn{2}{c}{ Parameter Combination } & Time & MRR & HPc & HPm & \\
& $\mathrm{F}$ & $\mathrm{Vr}$ & $\mathrm{a}$ & $(\mathrm{min})$ & $\mathrm{mm} 3 / \mathrm{min}$ & $\mathrm{kW}$ & $\mathrm{kW}$ & $\mathrm{kWh}$ \\
\hline 1 & 592 & 5000 & 1 & 459.08 & 296 & 0.013 & 0.022 & 0.1718 \\
2 & 592 & 7500 & 2 & 2229.26 & 592 & 0.027 & 0.045 & 0.1716 \\
3 & 592 & 10000 & 3 & 151.65 & 888 & 0.04 & 0.067 & 0.1703 \\
4 & 1184 & 5000 & 2 & 119.65 & 1184 & 0.54 & 0.09 & 0.1791 \\
5 & 1184 & 7500 & 3 & 75.56 & 1776 & 0.081 & 0.135 & 0.1697 \\
6 & 1184 & 10000 & 1 & 236.2 & 592 & 0.027 & 0.045 & 0.1768 \\
7 & 1776 & 5000 & 3 & 51.55 & 2664 & 0.121 & 0.202 & 0.1736 \\
8 & 1776 & 7500 & 1 & 155.95 & 888 & 0.04 & 0.067 & 0.1751 \\
9 & 1776 & 10000 & 2 & 77.28 & 1776 & 0.081 & 0.135 & 0.1735 \\
\hline
\end{tabular}

Table 10 shows the parameter value of the contour process. From table 11 it can be concluded that the optimal power response value in the contour process is in the $5^{\text {th }}$ combination with feed rate value $1184 \mathrm{~mm} /$ minute (level II), re-track rate $7500 \mathrm{~mm} /$ minute (level II), and depth of cut $3 \mathrm{~mm}$ (level III) with power response value $0,1697 \mathrm{kWh}$ and time 75,56 minutes.

TABLE 12.

PARAMETER VALUE OF SURFACE FINISH CONTOUR

\begin{tabular}{cccccc}
\hline Factor Name & Code & Level I & Level II & Level II & Unit \\
\hline Feed Rate & F & 1110 & 2220 & 331 & $\mathrm{~mm} / \mathrm{min}$ \\
Retrack Rate & $\mathrm{Vr}$ & 5000 & 7500 & 10000 & $\mathrm{~mm} / \mathrm{min}$ \\
Depth of Cut & $\mathrm{a}$ & 1 & 2 & 3 & $\mathrm{~mm}$ \\
\hline
\end{tabular}

TABLE 13.

POWER RESPONSES OF SURFACE ROUGH POCKET

\begin{tabular}{ccccccccc}
\hline No & \multicolumn{2}{c}{ Parameter Combination } & Time & MRR & HPc & HPm & kWh \\
& $\mathrm{F}$ & $\mathrm{Vr}$ & $\mathrm{a}$ & $(\mathrm{min})$ & $\mathrm{mm} 3 / \mathrm{min}$ & $\mathrm{kW}$ & $\mathrm{kW}$ & \\
\hline 1 & 1110 & 5000 & 0.5 & 536.55 & 277.5 & 0.013 & 0.021 & 0.01883 \\
2 & 1110 & 7500 & 0.75 & 236.02 & 416.25 & 0.019 & 0.032 & 0.1242 \\
3 & 1110 & 10000 & 1 & 175.66 & 555 & 0.025 & 0.042 & 0.1233 \\
4 & 2220 & 5000 & 0.75 & 182.15 & 832.5 & 0.038 & 0.063 & 0.1917 \\
5 & 2220 & 7500 & 1 & 120.33 & 1110 & 0.051 & 0.084 & 0.1689 \\
6 & 2220 & 10000 & 0.5 & 369.2 & 555 & 0.025 & 0.042 & 0.2591 \\
7 & 3331 & 5000 & 1 & 102.53 & 1665.5 & 0.076 & 0.126 & 0.2159 \\
8 & 3331 & 7500 & 0.5 & 315.8 & 832.75 & 0.038 & 0.063 & 0.3325 \\
9 & 3331 & 10000 & 0.75 & 152.86 & 1249.125 & 0.057 & 0.095 & 0.2414 \\
\hline
\end{tabular}

Table 12 shows the parameter value of the surface finish contour process. From table 13 it can be concluded that the optimal power response value in the surface finish contour process in the $3^{\text {rd }}$ combination with feed rate value $1110 \mathrm{~mm} /$ minute (level I), re-track rate $10000 \mathrm{~mm} /$ minute (level III), and depth of cut $1 \mathrm{~mm}$ ( level I). 
International Journal of Marine Engineering Innovation and Research, Vol. 5(1), Mar. 2020. 20-33 (pISSN:2541-5972, eISSN: 2548-1479)

TABLE 14

PARAMETER VALUE OF SURFACE FINISH PARALLEL

\begin{tabular}{cccccc}
\hline Factor Name & Code & Level I & Level II & Level II & Unit \\
\hline Feed Rate & F & 529 & 1184 & 1776 & $\mathrm{~mm} / \mathrm{min}$ \\
Retrack Rate & Vr & 5000 & 7500 & 10000 & $\mathrm{~mm} / \mathrm{min}$ \\
Depth of Cut & a & 1 & 2 & 3 & $\mathrm{~mm}$ \\
\hline
\end{tabular}

TABLE 15.

POWER RESPONSES OF SURFACE FINISH PARALLEL

\begin{tabular}{ccccccccc}
\hline No & \multicolumn{2}{c}{ Parameter Combination } & Time & MRR & HPc & HPm & kWh \\
& $\mathrm{F}$ & $\mathrm{Vr}$ & $\mathrm{a}$ & $(\mathrm{min})$ & $\mathrm{mm} 3 / \mathrm{min}$ & $\mathrm{kW}$ & $\mathrm{kW}$ & \\
\hline 1 & 355 & 5000 & 1 & 35.96 & 4260 & 0.194 & 0.323 & 0.1937 \\
2 & 355 & 7500 & 2 & 18.2 & 8520 & 0.388 & 0.646 & 0.19607 \\
3 & 355 & 10000 & 3 & 12.18 & 12780 & 0.582 & 0.97 & 0.19683 \\
4 & 711 & 5000 & 2 & 17.96 & 17064 & 0.777 & 1.295 & 0.38752 \\
5 & 711 & 7500 & 3 & 9.08 & 25596 & 1.165 & 1.942 & 0.29388 \\
6 & 711 & 10000 & 1 & 16.03 & 8532 & 0.388 & 0.647 & 0.17294 \\
7 & 1066 & 5000 & 3 & 4.1 & 38376 & 1.747 & 2.912 & 0.19895 \\
8 & 1066 & 7500 & 1 & 12.05 & 12792 & 0.582 & 0.971 & 0.19491 \\
9 & 1066 & 10000 & 2 & 6.08 & 25584 & 1.165 & 1.941 & 0.19669 \\
\hline
\end{tabular}

Table 14 shows the parameter value of the surface to finish the parallel process. From table 15 it can be concluded that the optimal power response value in the surface finish parallel process is in the $6^{\text {th }}$ combination with feed rate value of $711 \mathrm{~mm} /$ minute (level II), re-track rate $10000 \mathrm{~mm} / \mathrm{minute}$ (level III), and depth of cut $1 \mathrm{~mm}$ (level 1I) with a power response value of $0,17294 \mathrm{kWh}$ and processing time 16,03 minutes.

TABLE 16

PARAMETER VALUE OF FACING (BOTTOM)

\begin{tabular}{cccccc}
\hline Factor Name & Code & Level I & Level II & Level II & Unit \\
\hline Feed Rate & $\mathrm{F}$ & 395 & 592 & 790 & $\mathrm{~mm} / \mathrm{min}$ \\
Retrack Rate & $\mathrm{Vr}$ & 5000 & 7500 & 10000 & $\mathrm{~mm} / \mathrm{min}$ \\
Depth of Cut & $\mathrm{a}$ & 1 & 1.5 & 2 & $\mathrm{~mm}$ \\
\hline
\end{tabular}

TABLE 17.

POWER RESPONSES OF FACING (BOTTOM)

\begin{tabular}{|c|c|c|c|c|c|c|c|c|}
\hline \multirow{2}{*}{ No } & \multicolumn{3}{|c|}{ Parameter Combination } & \multirow{2}{*}{$\begin{array}{l}\text { Time } \\
(\min )\end{array}$} & \multirow{2}{*}{$\begin{array}{c}\mathrm{MRR} \\
\mathrm{mm} 3 / \mathrm{min}\end{array}$} & \multirow{2}{*}{$\begin{array}{l}\mathrm{HPc} \\
\mathrm{kW}\end{array}$} & \multirow{2}{*}{$\begin{array}{c}\mathrm{HPm} \\
\mathrm{kW}\end{array}$} & \multirow{2}{*}{$\mathrm{kWh}$} \\
\hline & $\mathrm{F}$ & $\mathrm{Vr}$ & $\mathrm{a}$ & & & & & \\
\hline 1 & 395 & 5000 & 1 & 91.02 & 4740 & 0.216 & 0.36 & 0.32732 \\
\hline 2 & 395 & 7500 & 1.5 & 60.65 & 7110 & 0.324 & 0.539 & 0.32716 \\
\hline 3 & 395 & 10000 & 2 & 53.25 & 9480 & 0.432 & 0.719 & 0.38299 \\
\hline 4 & 592 & 5000 & 1.5 & 40.56 & 10656 & 0.485 & 0.808 & 0.32791 \\
\hline 5 & 592 & 7500 & 2 & 36.42 & 14208 & 0.647 & 1.078 & 0.39258 \\
\hline 6 & 592 & 10000 & 1 & 60.84 & 7104 & 0.323 & 0.539 & 0.32791 \\
\hline 7 & 790 & 5000 & 2 & 30.46 & 18960 & 0.863 & 1.438 & 0.43816 \\
\hline 8 & 790 & 7500 & 1 & 44.62 & 9480 & 0.432 & 0.719 & 0.32092 \\
\hline 9 & 790 & 10000 & 1.5 & 34.23 & 14220 & 0.647 & 1.079 & 0.36929 \\
\hline
\end{tabular}

Table 16 shows the parameter value of the facing process. From table 17 it can be concluded that the optimal power response value in the facing process is in the $8^{\text {th }}$ combination with feed rate value $790 \mathrm{~mm} /$ minute (level III), re-track rate $7500 \mathrm{~mm} /$ minute (level II), and depth of cut $1 \mathrm{~mm}$ (level I) with power response value $0,32092 \mathrm{kWh}$ and processing time 44,62 minutes. 
International Journal of Marine Engineering Innovation and Research, Vol. 5(1), Mar. 2020. 20-33 (pISSN:2541-5972, eISSN: 2548-1479)

TABLE 18

PARAMETER VALUE OF CONTOUR (BOTTOM)

\begin{tabular}{cccccc}
\hline Factor Name & Code & Level I & Level II & Level II & Unit \\
\hline Feed Rate & F & 592 & 1184 & 1776 & $\mathrm{~mm} / \mathrm{min}$ \\
Retrack Rate & Vr & 5000 & 7500 & 10000 & $\mathrm{~mm} / \mathrm{min}$ \\
Depth of Cut & $\mathrm{a}$ & 1 & 2 & 3 & $\mathrm{~mm}$ \\
\hline
\end{tabular}

TABLE 19

POWER RESPONSES OF CONTOUR (BOTTOM)

\begin{tabular}{ccccccccc}
\hline \multirow{2}{*}{ No } & \multicolumn{2}{c}{ Parameter Combination } & Time & MRR & HPc & HPm & kWh \\
& $\mathrm{F}$ & $\mathrm{Vr}$ & $\mathrm{a}$ & $(\mathrm{min})$ & $\mathrm{mm} 3 / \mathrm{min}$ & $\mathrm{kW}$ & $\mathrm{kW}$ & \\
\hline 1 & 592 & 5000 & 1 & 475.63 & 296 & 0.013 & 0.022 & 0.10681 \\
2 & 592 & 7500 & 2 & 238.16 & 592 & 0.027 & 0.045 & 0.10697 \\
3 & 592 & 10000 & 3 & 162.36 & 888 & 0.04 & 0.067 & 0.10938 \\
4 & 1184 & 5000 & 2 & 120.16 & 1184 & 0.054 & 0.09 & 0.10794 \\
5 & 1184 & 7500 & 3 & 92.03 & 1776 & 0.081 & 0.135 & 0.124 \\
6 & 1184 & 10000 & 1 & 239.62 & 592 & 0.027 & 0.045 & 0.10762 \\
7 & 1776 & 5000 & 3 & 65.25 & 2664 & 0.121 & 0.202 & 0.13188 \\
8 & 1776 & 7500 & 1 & 156.1 & 888 & 0.04 & 0.067 & 0.10517 \\
9 & 1776 & 10000 & 2 & 90.83 & 1776 & 0.081 & 0.135 & 0.12239 \\
\hline
\end{tabular}

Table 18 shows the parameter value of the contour (level III), re-track rate $7500 \mathrm{~mm} /$ minute (level II), and process. From table 19 it can be concluded that the depth of cut $1 \mathrm{~mm}$ (level I) with power response value of optimal power response value in contour process is in the $0,10517 \mathrm{kWh}$ and processing time 156,1 minutes $8^{\text {th }}$ combination with feed rate value $1776 \mathrm{~mm} /$ minute

TABLE 20.

PARAMETER VALUE OF SURFACE ROUGH POCKET (BOTTOM)

\begin{tabular}{cccccc}
\hline Factor Name & Code & Level I & Level II & Level II & Unit \\
\hline Feed Rate & $\mathrm{F}$ & 592 & 1184 & 1776 & $\mathrm{~mm} / \mathrm{min}$ \\
Retrack Rate & $\mathrm{Vr}$ & 5000 & 7500 & 10000 & $\mathrm{~mm} / \mathrm{min}$ \\
Depth of Cut & $\mathrm{a}$ & 1 & 2 & 3 & $\mathrm{~mm}$ \\
\hline
\end{tabular}

TABLE 21.

POWER RESPONSES OF SURFACE ROUGH POCKET (BOTTOM)

\begin{tabular}{|c|c|c|c|c|c|c|c|c|}
\hline \multirow{2}{*}{ No } & \multicolumn{3}{|c|}{ Parameter Combination } & \multirow{2}{*}{$\begin{array}{l}\text { Time } \\
(\min )\end{array}$} & \multirow{2}{*}{$\begin{array}{c}\mathrm{MRR} \\
\mathrm{mm} 3 / \mathrm{min}\end{array}$} & \multirow{2}{*}{$\begin{array}{l}\mathrm{HPc} \\
\mathrm{kW}\end{array}$} & \multirow{2}{*}{$\begin{array}{l}\mathrm{HPm} \\
\mathrm{kW}\end{array}$} & \multirow{2}{*}{$\mathrm{kWh}$} \\
\hline & $\mathrm{F}$ & $\mathrm{Vr}$ & $\mathrm{a}$ & & & & & \\
\hline 1 & 592 & 5000 & 1 & 2276.66 & 7104 & 0.323 & 0.539 & 12.2705 \\
\hline 2 & 592 & 7500 & 2 & 1172.25 & 14208 & 0.647 & 1.078 & 12.6361 \\
\hline 3 & 592 & 10000 & 3 & 816.46 & 21312 & 0.97 & 1.617 & 13.2014 \\
\hline 4 & 1184 & 5000 & 2 & 582.26 & 28416 & 1.294 & 2.156 & 12.5528 \\
\hline 5 & 1184 & 7500 & 3 & 409.2 & 42624 & 1.94 & 3.234 & 13.2327 \\
\hline 6 & 1184 & 10000 & 1 & 1139.2 & 14208 & 0.647 & 1.078 & 12.2798 \\
\hline 7 & 1776 & 5000 & 3 & 273.45 & 63936 & 2.91 & 4.851 & 13.2643 \\
\hline 8 & 1776 & 7500 & 1 & 756.02 & 21312 & 0.97 & 1.617 & 12.2241 \\
\hline 9 & 1776 & 10000 & 2 & 388.96 & 42624 & 1.94 & 3.234 & 12.5782 \\
\hline
\end{tabular}

Table 20 shows the parameter value of the surface rough pocket process. From table 21 it can be concluded that the optimal power response value in the surface rough pocket process is in the $8^{\text {th }}$ combination with feed rate value $1776 \mathrm{~mm} /$ minute (level III), re-track rate 7500 $\mathrm{mm} /$ minute (level II), and depth of cut $1 \mathrm{~mm}$ (level I) with power response value $12,2241 \mathrm{kWh}$ and processing time 756,02 minutes. 
International Journal of Marine Engineering Innovation and Research, Vol. 5(1), Mar. 2020. 20-33 (pISSN:2541-5972, eISSN: 2548-1479)

TABLE 22

PARAMETER VALUE OF SURFACE FINISH CONTOUR

\begin{tabular}{cccccc}
\hline Factor Name & Code & Level I & Level II & Level II & Unit \\
\hline Feed Rate & F & 1110 & 2220 & 3331 & $\mathrm{~mm} / \mathrm{min}$ \\
Retrack Rate & Vr & 5000 & 7500 & 10000 & $\mathrm{~mm} / \mathrm{min}$ \\
Depth of Cut & a & 1 & 2 & 3 & $\mathrm{~mm}$ \\
\hline
\end{tabular}

TABLE 23

POWER RESPONSES OF SURFACE FINISH CONTOUR

\begin{tabular}{ccccccccc}
\hline \multirow{2}{*}{ No } & \multicolumn{2}{c}{ Parameter Combination } & Time & MRR & HPc & HPm & kWh \\
& $\mathrm{F}$ & $\mathrm{Vr}$ & $\mathrm{a}$ & $(\mathrm{min})$ & $\mathrm{mm} 3 / \mathrm{min}$ & $\mathrm{kW}$ & $\mathrm{kW}$ & \\
\hline 1 & 1110 & 5000 & 0.5 & 551.26 & 277.5 & 0.013 & 0.021 & 0.11606 \\
2 & 1110 & 7500 & 0.75 & 276.05 & 416.25 & 0.019 & 0.032 & 0.08718 \\
3 & 1110 & 10000 & 1 & 185.86 & 555 & 0.025 & 0.042 & 0.07826 \\
4 & 2220 & 5000 & 0.75 & 190.42 & 832.5 & 0.038 & 0.063 & 0.12027 \\
5 & 2220 & 7500 & 1 & 128.66 & 1110 & 0.051 & 0.084 & 0.10835 \\
6 & 2220 & 10000 & 0.5 & 380.55 & 555 & 0.025 & 0.042 & 0.16024 \\
7 & 3331 & 5000 & 1 & 109.6 & 1665.5 & 0.079 & 0.126 & 0.13849 \\
8 & 3331 & 7500 & 0.5 & 323.6 & 832.75 & 0.038 & 0.063 & 0.20445 \\
9 & 3331 & 10000 & 0.75 & 161.86 & 1249.125 & 0.057 & 0.095 & 0.15339 \\
\hline
\end{tabular}

Table 22 shows the parameter value of the surface finish contour process. From table 23 it can be concluded that the optimal power response values in the process of surface finish contour are in the $3^{\text {rd }}$ combination with feed rate value of $1110 \mathrm{~mm} /$ minute (level I), re-track rate $10000 \mathrm{~mm} /$ minute (level III), and depth of cut $1 \mathrm{~mm}$ (level I) with power response value $0.07826 \mathrm{kWh}$ and time $\quad 185,86$ minutes.

TABLE 24

PARAMETER VALUE OF SURFACE FINISH PARALEL

\begin{tabular}{cccccc}
\hline Factor Name & Code & Level I & Level II & Level II & Unit \\
\hline Feed Rate & F & 592 & 1184 & 1776 & $\mathrm{~mm} / \mathrm{min}$ \\
Retrack Rate & $\mathrm{Vr}$ & 5000 & 7500 & 10000 & $\mathrm{~mm} / \mathrm{min}$ \\
Depth of Cut & $\mathrm{a}$ & 1 & 2 & 3 & $\mathrm{~mm}$ \\
\hline
\end{tabular}

TABLE 25

POWER RESPONSES OF SURFACE FINISH PARALLEL

\begin{tabular}{ccccccccc}
\hline \multirow{6}{*}{ No } & \multicolumn{2}{c}{ Parameter Combination } & Time & MRR & HPc & HPm & kWh \\
& $\mathrm{F}$ & $\mathrm{Vr}$ & $\mathrm{a}$ & $(\mathrm{min})$ & $\mathrm{mm} 3 / \mathrm{min}$ & $\mathrm{kW}$ & $\mathrm{kW}$ & \\
\hline 1 & 355 & 5000 & 1 & 563.26 & 4260 & 0.194 & 0.323 & 1.82045 \\
2 & 355 & 7500 & 2 & 287.98 & 8520 & 0.388 & 0.646 & 1.86149 \\
3 & 355 & 10000 & 3 & 189.7 & 12780 & 0.582 & 0.97 & 1.83932 \\
4 & 711 & 5000 & 2 & 146.02 & 17064 & 0.777 & 1.295 & 1.8904 \\
5 & 711 & 7500 & 3 & 102.2 & 25596 & 1.165 & 0.942 & 1.98464 \\
6 & 711 & 10000 & 1 & 290.6 & 8532 & 0.388 & 0.647 & 1.88108 \\
7 & 1066 & 5000 & 3 & 78.52 & 38376 & 1.747 & 0.2912 & 2.28612 \\
8 & 1066 & 7500 & 1 & 187.05 & 12792 & 0.582 & 0.971 & 1.81533 \\
9 & 1066 & 10000 & 2 & 112.03 & 25584 & 1.165 & 1.941 & 2.17452 \\
\hline
\end{tabular}

Table 24 shows the parameter value of the surface finish the parallel process. From table 25 it can be concluded that the optimal power response value in the surface finish parallel process is in the $8^{\text {th }}$ combination with feedrate value $1066 \mathrm{~mm} /$ minute (level III), ret-rack rate $7500 \mathrm{~mm} /$ minute (level II), and depth of cut $1 \mathrm{~mm}$ (level I) with power response value $1,81533 \mathrm{kWh}$ and processing time 187,05 minutes. 
TABLE 26.

TOTAL PROCESSING TIME AND POWER CUTTING REQUIREMENT

\begin{tabular}{cccc}
\hline NO & Process & Time (minute) & Power (kWh) \\
\hline 1 & Facing & 20 & 1,3474 \\
2 & Contour & 76,56 & 0,1697 \\
3 & Surface Rough Pocket & 440,92 & 7,9214 \\
4 & Surface Finish Contour & 175,66 & 0,1233 \\
5 & Surface Finish Parallel & 16,03 & 0,17294 \\
6 & Facing & 44,62 & 0,32092 \\
7 & Contour & 156,1 & 0,10517 \\
8 & Surface Rough Pocket & 756,02 & 12,2241 \\
9 & Surface Finish Contour & 185,86 & 0,07826 \\
10 & Surface Finish Parallel & 187,05 & 1,81533 \\
\hline
\end{tabular}

\section{E. Analysis of Variance (ANOVA)}

From the results of simulations that have been carried out then proceed with analysis using variance analysis according to parameter factors, level, and processing of data based on ANOVA statistics to determine the effect of each factor variable (feed rate, re-track rate and depth of cut) on the response variable (machining process power). The variance analysis process software 18 .

Results of Analysis of Variance:

TABLE 27.

ANALYSIS OF VARIANCE FACING PROCESS

\begin{tabular}{ccccc}
\hline Source & DF & Adj SS & F-Value & P-Value \\
\hline Feed Rate & 2 & 898.02 & 41.81 & 0,023 \\
Retrack Rate & 2 & 48.25 & 2.25 & 0,308 \\
Depth of Cut & 2 & 877.23 & 40.85 & 0,024 \\
Error & 2 & 21.48 & - & - \\
Total & 8 & 1844.98 & - & - \\
\hline
\end{tabular}

Based on Table 27, it can conclude several things. If the value of $\mathrm{P}>0.05$ means that there are no significant factors that influence the power response. In the facing feed rate process, and the depth of cut has a value of $\mathrm{P}$ $<0.05$ means that it significantly affects the power response. In the facing process the greatest $F$ value of the three factors in the experiment is feed rate, which is 41,81 which means that feed rate has the greatest influence on the power response, then the second depth of cut with $\mathrm{F}=40,85$ and the last re-track rate with $\mathrm{F}=$ 2,25 .

TABLE 28

ANALYSIS OF VARIANCE CONTOUR PROCESS

\begin{tabular}{ccccc}
\hline Source & DF & Adj SS & F-Value & P-Value \\
\hline Feed Rate & 2 & 55188 & 9.71 & 0,093 \\
Retrack Rate & 2 & 6225 & 1.09 & 0,477 \\
Depth of Cut & 2 & 58902 & 10.36 & 0,088 \\
Error & 2 & 5685 & - & - \\
Total & 8 & 126001 & - & - \\
\hline
\end{tabular}

Based on Table 28 it can conclude several things. If the value of $\mathrm{P}>0.05$ means that there are no factors that most influence the power response. In the contour, all factors have a value of $\mathrm{P}>0.05$, which means there are no factors that significantly affect the power response. In the contour process, the greatest $F$ value of the three factors in the experiment is a depth of cut, which is 10.36, which means that depth of cut has the greatest influence on the power response, then the second is feed rate with $\mathrm{F}=9.71$ and the last re-track rate with $\mathrm{F}=1.09$. 
TABLE 29.

ANALYSIS OF SURFACE ROUGH POCKET

\begin{tabular}{ccccc}
\hline Source & DF & Adj SS & F-Value & P-Value \\
\hline Feed Rate & 2 & 304222 & 9,61 & 0,094 \\
Retrack Rate & 2 & 40199 & 1,27 & 0,441 \\
Depth of Cut & 2 & 237748 & 7,51 & 0,117 \\
Error & 2 & 31653 & - & - \\
Total & 8 & 613821 & - & - \\
\hline
\end{tabular}

Based on table 29, it can conclude several things. If the value of $\mathrm{P}>0.05$ means that there are no significant factors that influence the power response. In the surface rough pocket process, all factors have a value of $\mathrm{P}>0.05$, which means there are no factors that significantly influence the power response. In the surface rough pocket process the greatest $F$ value of the three factors in the experiment is the feed rate which is 9.61 meaning that the feed rate has the greatest influence on the power response, then the second is the depth of cut with the value $F=7.51$ and the last re-track rate with the value $F$ $=1.27$.

TABLE 30

ANALYSIS OF VARIANCE SURFACE FINISH CONTOUR

\begin{tabular}{ccccc}
\hline Source & DF & Adj SS & F-Value & P-Value \\
\hline Feed Rate & 2 & 25415 & 7,60 & 0,116 \\
Retrack Rate & 2 & 4237 & 1,27 & 0,441 \\
Depth of Cut & 2 & 125590 & 37,55 & 0,026 \\
Error & 2 & 3344 & - & - \\
Total & 8 & 158587 & - & - \\
\hline
\end{tabular}

Based on Table 30it can conclude several things. If greatest $F$ value of the three factors in the experiment is the value of $\mathrm{P}>0.05$ means that there are no significant factors that influence the power response. In the surface finish contour process, the depth of cut has a value of $\mathrm{P}$ $<0.05$ means that it significantly affects the power the depth of cut, which is 37,55 which means that depth of cut has the greatest influence on the power response, then the second feed rate with $\mathrm{F}=7,60$ and the last reresponse. In the surface finish contour process, the track rate with $\mathrm{F}=1,27$

TABLE 31

ANALYSIS OF VARIANCE SURFACE FINISH PARALLEL

\begin{tabular}{ccccc}
\hline Source & DF & Adj SS & F-Value & P-Value \\
\hline Feed Rate & 2 & 324,61 & 11,31 & 0,081 \\
Retrack Rate & 2 & 104,20 & 3,63 & 0,216 \\
Depth of Cut & 2 & 250,70 & 8,74 & 0,103 \\
Error & 2 & 28,69 & - & - \\
Total & 8 & 708,21 & - & - \\
\hline
\end{tabular}

Based on Table 31, it can conclude several things. If the value of $\mathrm{P}>0.05$ means that there are no significant factors that influence the power response. In the process of surface finish parallel feed rate has a value of $\mathrm{P}<0.05$, which means that it significantly affects the power response. In the process of surface finish parallel, the highest value of $F$ from the three factors in the experiment is feed rate which is 11.31 , meaning that feed rate has the greatest influence on power response, then the second depth of cut with $\mathrm{F}=8.74$ and the last retrack rate with $\mathrm{F}=3.63$. 
TABLE 32.

ANALYSIS OF VARIANCE FACING (BOTTOM)

\begin{tabular}{ccccc}
\hline Source & DF & Adj SS & F-Value & P-Value \\
\hline Feed Rate & 2 & 324,61 & 11,31 & 0,081 \\
Retrack Rate & 2 & 104,20 & 3,63 & 0,216 \\
Depth of Cut & 2 & 250,70 & 8,74 & 0,103 \\
Error & 2 & 28,69 & - & - \\
Total & 8 & 708,21 & - & - \\
\hline
\end{tabular}

Based on Table 32, it can conclude several things. If the value of $\mathrm{P}>0.05$ means that there are no factors that most influence the power response. In the facing, all factors have a value of $\mathrm{P}>0.05$, which means there are no factors that significantly affect the power response. In the facing process, the greatest $\mathrm{F}$ value of the three factors in the experiment is feed rate, which is 11.31 , which means that feed rate has the greatest influence on the power response, then the second is a depth of cut with $\mathrm{F}=8.74$ and the last re-track rate with $\mathrm{F}=3.63$.

TABLE 33.

ANALYSIS OF VARIANCE CONTOUR

\begin{tabular}{ccccc}
\hline Source & DF & Adj SS & F-Value & P-Value \\
\hline Feed Rate & 2 & 57514 & 7,13 & 0,123 \\
Retrack Rate & 2 & 6542 & 0,81 & 0,552 \\
Depth of Cut & 2 & 55490 & 6,88 & 0,127 \\
Error & 2 & 8064 & & - \\
Total & 8 & 127610 & - & - \\
\hline
\end{tabular}

Based on Table 33, it can conclude several of things. If the value of $\mathrm{P}>0.05$ means that there are no significant factors that influence the power response. In the contour process, all factors have a value of $\mathrm{P}>0.05$, which means there are no factors that significantly affect the power response. In the contour process, the highest value of the three factors in the experiment is the feed rate, which is 7.13. It means that feed rate has the most influence on the power response, then the second depth of cut with $\mathrm{F}$ $=6.88$ and the last re-track rate with $\mathrm{F}=0.81$.

Based on Table 34, it can conclude several of things. If the value of $\mathrm{P}>0.05$ means that there are no significant factors that influence the power response. In the surface rough pocket process, all factors have a value of $\mathrm{P}>0.05$, which means there are no factors that significantly influence the power response. In the surface rough pocket process, the highest $F$ value of the three factors in the experiment is feed rate, which is 10.36 , which means that feed rate has the most influence on the power response, then the second depth of cut with $\mathrm{F}=9.18$ and the last re-track rate with $\mathrm{F}=0.98$.

TABLE 34.

ANALYSIS OF SURFACE ROUGH POCKET

\begin{tabular}{ccccc}
\hline Source & DF & Adj SS & F-Value & P-Value \\
\hline Feed Rate & 2 & 1463258 & 10,36 & 0,088 \\
Retrack Rate & 2 & 139163 & 0,98 & 0,504 \\
Depth of Cut & 2 & 1297038 & 9,18 & 0,098 \\
Error & 2 & 141290 & - & - \\
Total & 8 & 3040750 & - & - \\
\hline
\end{tabular}


TABLE 35 .

ANALYSIS OF VARIANCE SURFACE FINISH CONTOUR

\begin{tabular}{ccccc}
\hline Source & DF & Adj SS & F-Value & P-Value \\
\hline Feed Rate & 2 & 31562 & 9,39 & 0,096 \\
Retrack Rate & 2 & 3361 & 1,00 & 0,500 \\
Depth of Cut & 2 & 125108 & 37,23 & 0,026 \\
Error & 2 & 3361 & - & - \\
Total & 8 & 163392 & - & - \\
\hline
\end{tabular}

Based on Table 35 it can conclude several of things. If the value of $\mathrm{P}>0.05$ means that there are no significant factors that influence the power response. In the process of surface finish contour depth of cut has a value of $\mathrm{P}$ $<0.05$ which means that it significantly affects the power response. In the process of surface finish contour, thehighest value of $\mathrm{F}$ from the three factors in the experiment is depth of cut which is 37.23 , meaning that depth of cut has the greatest influence on power response, then the second feed rate with $\mathrm{F}=9.39$ and the last re-track rate with $\mathrm{F}=1.00$.

TABLE 36

ANALYSIS OF VARIANCE SURFACE FINISH PARALLEL

\begin{tabular}{ccccc}
\hline Source & DF & Adj SS & F-Value & P-Value \\
\hline Feed Rate & 2 & 79793 & 7,37 & 0,119 \\
Retrack Rate & 2 & 9197 & 0,85 & 0,541 \\
Depth of Cut & 2 & 80589 & 7,45 & 0,118 \\
Error & 2 & 10825 & - & - \\
Total & 8 & 180404 & - & - \\
\hline
\end{tabular}

Based on Table 36, it can conclude a number of things. If the value of $\mathrm{P}>0.05$ means that there are no significant factors that influence the power response. In the process of surface finish parallel, all factors have a value of $\mathrm{P}$ $>0.05$, meaning that there are no factors that significantly affect the power response. In the surface finish parallel process, the highest $F$ value of the three factors in the experiment is the depth of cut, which is 7.45 . It means that the depth of cut has the greatest influence on the power response, then the second is feed rate with the value $\mathrm{F}=7.37$ and re-track rate with $\mathrm{F}=0.85$.

\section{CONCLUSION}

\section{A. Conclusion}

Based on the simulation results of the production of dies propeller in the MasterCam software with several combinations of machining parameters, obtained several conclusions to answer the formulation of the problem on this paper, as follows:

1. In the dies propeller production simulation, five steps are performed; there are facing, contour, rough surface pocket, surface finish contour and, surface finish parallel. That 5 processes are used for both top and bottom dies.
2. From the simulation results that have been carried out, the total production time of dies propeller is obtained, which is 729,17 minutes with power consumption $9.734 \mathrm{kWh}$ for top dies and 1329,65 minutes with power consumption $14,54 \mathrm{kWh}$ for bottom dies. 


\section{REFERENCES}

[1] Utomo, Cahyo. 2017. Perancangan dan Pembuatan Dies Permanent Mold Pengecoran Logam Dengan Material Besi Cor Ductile. Surakarta: Universitas Muhammadiyah Surakarta

[2] Firdaus. 2009. Rancang Bangun Cetakan Permanen (Permanent Mold) Untuk Pembuatan Pulley Aluminium. Palembang: Politeknik Negeri Sriwijaya

[3] I G.N.K. Yudhyadi, T. R. 2016. Optimasi Parameter Permesinan Terhadap Waktu Proses Pada Pemrograman CNC Milling dengan Berbasis CAD/CAM. Dinamika Teknik Mesin, VI (1), 38-50.

[4] Rochim, Taufiq. 2002. Optimasi Proses Pemesinan. Bandung: Institut Teknologi Bandung

[5] Supriyanta. 2018. Desain Dan Proses Pembuatan Cetakan Permanen Dengan Material Logam Besi Cor Kelabu Hasil Coran Pasir Co2 Untuk Proses Pembuatan Flange Dengan Material Kuningan. Surakarta: Universitas Muhammadiyah Surakarta.

[6] Adi Nugraha, Sasmita. 2018. Optimasi Desain Propeller Sea Water Pump Dengan Parameter Distribusi Blade Angle Pada Daerah Shroud Menggunakan Pendekatan Computational Fluid Dynamics (CFD). Surabaya: Institut Teknologi Sepuluh Nopember

[7] Wijanarka, B. 2013. Cadcam untuk Mesin Bubut dan Frais Menggunakan MasterCam 9 dan X3. Yogyakarta.

[8] Sumbodo, W. 2008. Teknik Produksi Mesin Industri Jilid 1. Jakarta. Direktorat Pembinaan Sekolah Menengah Kejuruan

[9] Kumbhar. 2015. Multi-objective Optimization of Machining Parameters in CNC End Milling of Stainless Steel 304. India: K.B.P. College of Engineering

[10] Atmadio, Nico. 2018. Optimasi Jalan Pahat dan Analisis Biaya Produksi Proses Permesinan CNC Lathe Pembuatan Piston
Master Cylinder Rem Sepeda Motor Yamaha Menggunakan CAM. Pekanbaru: Universitas Riau

[11] Daniel. (2009). Optimasi Parameter Pemesinan Proses Cnc Frais Terhadap Hasil Kekasaran Permukaan Dan Keausan Pahat Menggunakan Metode Taguchi. Semarang: Universitas Diponegoro

[12] Bagus Praetyo, A. 2015. Aplikasi Metode Taguchi Pada Optimasi Parameter Permesinan Terhadap Kekasaran Permukaan Dan Keausan Pahat Hss Pada Proses Bubut Material St 37. Kediri: Universitas Nusantara Persatuan Guru Republik Indonesia

[13] Hamdani, Ficky. 2014. Optimasi Pemesinan Pada Mesin Bubut Tipe M-300 Horrison Dengan Metode Optimasi Algoritma Genetika. Medan: Universitas Sumatera Utara

[14] Candra, Susila. 2009. Optimasi Proses Pemesinan Milling Fitur Pocket Material Baja Karbon Rendah Menggunakan Response Surface Methodology. Surabaya: Universitas Surabaya

[15] Anto,Edi. 2013. Optimasi Parameter Permesinan Terhadap Waktu Proses Pada Pemrograman CNC Turning. Semarang: Universitas Negeri Semarang

[16] Iskandar, W. 2016. Analisa Teoritis Kebutuhan Daya Mesin Bubut Gear Head Turret. Surakarta: Universitas Muhammadiyah Surakarta.

[17] Mosey, C.A. 2015. Perhitungan Waktu Dan Biaya Pada Proses Permesinan Benda Uji Tarik. Manado: Universitas Sam Ratulangi

[18] Ibnu Sabilillah, M. 2018. Perancangan dan Perhitungan Waktu Total Proses Produksi Blade Controllable Pitch Propeller Dengan CNC untuk Perencanaan Biaya Produksi. Surabya: Institut Teknologi Sepuluh Nopember.

[19] Van Hoten, Henry. 2012. Pemodelan Sistem Perhitungan Tarif Mesin Produksi Dengan Memperhitungkan Beban Langsung dan Tak Langsung. Bandung: Institut Teknologi Bandung. 